\title{
UNIVERSIDAD AUTÓNOMA VENEZOLANA: EXPECTATIVAS DE LAS AUTORIDADES RECTORALES
}

\author{
Norma M. Caira Tovar* \\ https://orcid.org/0000-0002-7629-8216 \\ José G. Sánchez Morles** \\ https://orcid.org/0000-0002-4619-6542
}

RECIBIDO: Febrero 2021 / ACEPTADO: Junio 2021 / PUBLICADO: Septiembre 2021

\begin{abstract}
Como citar: Caira Tovar, Norma; Sánchez Morles, José. (2021) Universidad Autónoma Venezolana: expectativas de las autoridades rectorales. Telos: revista de Estudios Interdisciplinarios en Ciencias Sociales, 23 (3), Venezuela. (Pp. 584-601).

DOI: www.doi.org/10.36390/telos233.06
\end{abstract}

\section{RESUMEN}

La tendencia de este milenio a observar la realidad desde un enfoque integral y humanista no ha obviado la actualidad universitaria, la cual ha manifestado limitaciones en su desenvolvimiento institucional. Este escenario conllevó a realizar un estudio que describiera las expectativas sobre la universidad autónoma venezolana desde la perspectiva de su gerencia, considerando para ello a las autoridades rectorales elegidas para el período 2008-2012 y que se han mantenido en sus cargos por más de diez años. La investigación fue enmarcada en el paradigma interpretativo, siguiendo los pasos del enfoque cualitativo, el método hermenéutico y el análisis de contenido, a través del uso de documentos institucionales de acceso público, emitidos por las autoridades de las universidades autónomas nacionales durante eventos realizados en fechas de relevancia (actos de grado, aniversarios institucionales y consejos universitarios extraordinarios), así como referencias teóricas de Albornoz (2013), Bolman y Deal (2017), Chiavenato (2017), Lussier y Achua (2016), Newstrom (2011), Robbins y Coulter (2018). Del análisis realizado emergieron las categorías construcción del futuro, incertidumbre y proyecto de país. Para la fiabilidad del estudio se realizó una triangulación de datos y de teoría (se contrastaron los contenidos de los documentos entre sí y con los referentes teóricos). Los hallazgos revelan que para las autoridades rectorales las expectativas constituyen un estado de esperanza, el cual requiere de unidad para encaminar esfuerzos en el proceso de recuperación de la universidad y de la reconstrucción del país. Se concluye que, desde la perspectiva rectoral, la gerencia de estas organizaciones cuenta con herramientas para superar su crisis institucional

\footnotetext{
* Doctora en Gerencia, MSc. Gerencia de Empresas. Sociólogo. Docente-Investigadora Titular Universidad del Zulia. MaracaiboVenezuela. E-mail: normacaira@gmail.com

** Doctor en Ciencias Gerenciales, MSc. Gerencia de Recursos Humanos, Msc. Gerencia de Proyectos I+D, Lic. Administración en Gerencia Industrial. Docente-Investigador Titular Universidad Privada Dr. Rafael Belloso Chacín. Maracaibo-Venezuela. E-mail: jgsanchezmorles@gmail.com
} 
(anterior a la aparición del Covid-19), recomendándose fortalecer su comunicación con la comunidad interna y externa a ellas, para lograr que la transparencia y fluidez informativa de sus planes les permitan conseguir el apoyo y la unificación de voluntades necesarios para recuperar su prestigio.

Palabras clave: Universidad autónoma venezolana; expectativas; construcción de futuro; incertidumbre; proyecto de país.

\section{Venezuelan Autonomous University: expectations of the rectoral authorities}

\section{ABSTRACT}

The trend of this millennium to observe reality from a comprehensive and humanistic approach has not ignored the current events in universities, which has manifested limitations in institutional development. This scenario led to a study that revealed the expectations about the Venezuelan autonomous university from the perspective of its management, considering the rectoral authorities elected to period 2008-2012 and who have remained in their positions for more than ten years. The research was framed in the interpretive paradigm, following the steps of the qualitative approach, the hermeneutical method, and the content analysis, through the use of institutional documents of public access, issued by the authorities of the autonomous national universities during events held on dates of relevance (graduation events, institutional anniversaries and extraordinary university councils), as well as theoretical references from Albornoz (2013), Bolman and Deal (2017), Chiavenato (2017), Lussier and Achua (2016), Newstrom (2011), Robbins and Coulter (2018). From the analysis performed, the categories construction of the future, uncertainty, and country project emerged. To the reliability of study a triangulation of data and theory was performed (the contents of the documents were contrasted by each other with the theoretical references). The findings reveal that expectations constitute a state of hope for the rectoral authorities, which requires unity to direct efforts in the university's recovery process and the country's reconstruction process. It is concluded that from the rectoral perspective, the organizational management has the necessary skills to overcome their institutional crisis (before the appearance of Covid-19), recommending strengthening their communication with the internal and external community, to achieve transparency and fluency information of their plans for to achieve the support and unification of wills necessary to regain its prestige.

Keywords: Venezuelan autonomous university; expectations; construction of the future; uncertainty; country project.

\section{Introducción}

Las autoridades rectorales de las universidades autónomas venezolanas tienen el desafío de tomar decisiones que permitan emprender iniciativas, convenios y adecuaciones viables como demostración de su capacidad para enfrentar limitaciones propias, así como por dar cabida al cumplimiento de su misión en un escenario caracterizado en este milenio por la tendencia de observar la realidad desde un enfoque integral y humanista. 
Una tendencia que invita a concebir la realidad mediante conocimientos renovados, junto a una capacidad de adaptación o promoción de cambios, con los cuales se abarquen esfuerzos por mejorar la calidad de vida del ser humano al considerar los aspectos socioculturales de cada institución y del contexto donde se inserta, incentivando el reconocimiento de los valores y saberes del hombre como vía para lograr el desarrollo y la sostenibilidad (Bokova, 2010).

Los aspectos anteriormente mencionados, remiten hacia la adopción de una direccionalidad cónsona con el desempeño esperado, una finalidad práctica que identifica a la organización como un medio para alcanzar un propósito y con posibilidad de adaptación a su entorno. En el caso universitario, estos elementos deberían incentivar a una ruptura con el modelo napoleónico que caracteriza a este sector, los cuales, desde la perspectiva de Drucker (2004), muestran a la universidad como una entidad rígida y menos flexible en comparación con las empresas, dada su predisposición a adherirse a conceptualizaciones, postulados y políticas gerenciales pasadas.

En tiempos más recientes, Albornoz (2013) y Acosta (2018), exponen que, desde las elecciones presidenciales del año 2012, las instituciones universitarias autónomas venezolanas están prácticamente paralizadas, debido a un proceso continuo de desatención del Estado, acompañado de un creciente retiro de personal, descuido en la actualización de sueldos y planes de seguridad social, debilitamiento de la libertad académica y de la integridad institucional.

De acuerdo con estos autores (Albornoz, 2013 y Acosta, 2018), son instituciones caracterizadas por su fuerte oposición hacia la política pública implementada hacia ellas, manifestada cada año a través de decisiones gubernamentales relacionadas con la disminución de la cantidad presupuestada para su funcionamiento, a pesar de que según el artículo 13 de la Ley de Universidades (Consejo Nacional de Universidades, 1970) les corresponde una partida anual mínima de $1.5 \%$ del total de rentas de la Ley de Presupuesto Nacional; como resultado de ello, desde el año 2018 estas instituciones se han declarado en una situación crítica que afecta su normal desenvolvimiento, un panorama catalogado como asfixia financiera (Aula Abierta Venezuela, 2020).

Por su parte, Ramírez (2020, p.71), considera que en la problemática del sector universitario incide un proceso de desinstitucionalización del subsistema de educación superior generado desde el Estado, unido a intentos de control político-partidista, todo lo cual ha agravado "...las tensiones con las universidades autónomas, lo que perjudica enormemente el cumplimiento de sus funciones tal como lo establece el artículo 2 de la Ley de Universidades vigente en cuanto a contribuir a orientar al país con su doctrina".

Al respecto, García-Arocha (2018), ha manifestado que "La universidad venezolana no se rinde ante la difícil situación por la que atraviesa nuestra nación, siempre ha estado presente", agregando Aular (2018a), que "no podemos quedarnos encerrados en ese concepto de crisis o de emergencia, es por eso que ha llegado la hora de reinventar la universidad, buscando alternativas que nos ayuden a salir adelante...". Por su parte, Primera (2017) caracterizó el escenario de las universidades como delicado, obligando a sus autoridades a seguir innovando en los procesos administrativos que brindan, conservando la calidad de los servicios estudiantiles y exponiendo la necesidad de asumir un rol activo en el desarrollo de la nación. 
De acuerdo con esta autoridad universitaria, el ámbito académico conlleva a mantenerse en una situación donde los términos crisis y universidad se manifiestan como un binomio, aspecto que durante las temporadas más conflictivas requiere convertir la reflexión y la mirada retrospectiva en elementos claves para superar esos momentos, agregando Aular (2019) que la universidad no volverá a cerrar sus puertas, haciendo referencia a la dificultad de transitar el momento crítico de estas instituciones, el cual percibe como reflejo de la propia crisis nacional.

En este sentido y en nombre del equipo rectoral, manifestó la obligación de realizar un llamado a la comunidad (autoridades, personal docente y de investigación, personal administrativo y obrero, estudiantes y egresados) para rescatar la universidad y tratar de evitar su naufragio ante un déficit presupuestario de $99 \%$ que compromete su funcionalidad; en tanto Divo, citada por Cárdenas (2019), se inclina hacia la necesidad de dar a conocer que las convenciones colectivas han sido burladas, a lo cual se une la insuficiencia presupuestaria para mantener la operatividad de estas instituciones.

A pesar de ello, para Pérez y Moreno (2017), las autoridades rectorales universitarias procuran mantener el cumplimiento de calidad de las funciones básicas de estas instituciones, enseñanza, investigación y extensión, contextualizadas con la problemática social, involucrando en esa tarea la misión y visión organizacional, lo que exterioriza la necesidad de contar con un direccionamiento capaz de permitir la sostenibilidad de la universidad del siglo XXI.

Una panorámica a la cual se agrega, según estos autores, el requerimiento de cumplir los criterios de eficacia establecidos en la Carta Universitaria Río 2014 (documento firmado como colorario de la Cumbre de Rectores realizada en Brasil durante ese año). Un documento donde se solicita mejorar la gestión y financiamiento de la educación superior en las instituciones iberoamericanas, acordando el establecimiento de estrategias referidas a la planificación y análisis de políticas, con la finalidad de lograr una cooperación entre ellas y los entes vinculados con su funcionamiento en cada país, un aspecto retomado por Marín (2020), como muestra de su vigencia.

La complejidad de este escenario se ve complementada por posturas similares a la planteada por Marcano (2017), quien añade que se hace prioritario lograr una transformación universitaria, la cual debe contar con dirigentes comprometidos con los cambios paradigmáticos demandados por la humanidad, una gerencia que esgrima el conocimiento hacia la construcción y el funcionamiento de un modelo productivo nacional en pro del desarrollo del país, en un esfuerzo sostenido para concebir y fundar una reforma de pensamiento conducente a la evolución de la sociedad.

Un escenario retomado por Sánchez y Cabrera (2021, p.107), quienes enfatizan la dificultad de definir la gerencia, "... por requerir criterios y orientaciones de carácter técnico y lo complejo de la dimensión humana, ambos dependen de experiencias y saberes que contribuyen en gran medida en la construcción a la misma". Considerando esta situación institucional (previa a la aparición del Covid-19) se desarrolló un estudio que permitió describir las expectativas sobre la universidad autónoma venezolana desde la perspectiva de su gerencia, involucrando aspectos no vinculados con la pandemia.

\section{Expectativas}

De acuerdo con Newstrom (2011) y Runes (2018), las expectativas son estados en los cuales se espera que un acontecimiento se concrete, denominándose de igual modo los motivos 
que soportan la creencia de un suceso, aludiendo a una probabilidad, suposición o esperanza razonable. A nivel psicológico, Warren (2013), las define como una actitud caracterizada por la tensión y la atención prospectiva, mientras que la fórmula motivacional de Vroom (retomada tanto por Lussier y Achua, 2016, como por Chiavenato, 2017), plantea que las personas pueden motivarse a cumplir sus tareas al creer en la recepción de una recompensa a cambio de su esfuerzo, definiendo el término expectativa como la percepción individual de la capacidad para alcanzar un objetivo.

En este sentido, Chiavenato (2017), Robbins y Coulter (2018), simplifican esa fórmula, expresando que este modelo consiste en una relación entre esfuerzo individual, desempeño particular, recompensas organizacionales y objetivos individuales con sus respectivos vínculos (esfuerzo-desempeño, desempeño-recompensa), complementados por los atractivos relacionados con la satisfacción de los logros alcanzados.

De conformidad con esta perspectiva, las personas tienden a actuar de manera particular basadas en la expectativa de que habrá un resultado posterior a su acción, la cual será de gran atractivo, añadiendo Hernández y Padilla (2019, p.226), que constituyen algo concreto, dado "...que no hacen referencia a un deseo, sino a un compromiso por alcanzar cierto nivel y suponen un plan realista para lograr esa meta". Una idea complementada por Dioses et al (2021, p.250), cuando destacan que "...las universidades deben preocuparse por la calidad educativa que imparten, realizando una óptima planificación, organización, ejecución, control y evaluación permanente, respondiendo mejor a las expectativas de los estudiantes".

\section{Construcción del futuro}

Este término se relaciona con pronóstico, definido por Robbins y Coulter (2018), como una técnica gerencial para analizar el entorno y definir las bases para realizar una planificación vinculada con un determinado pronóstico, entendida por estos autores como predicción de resultados. Desde su perspectiva, esa técnica puede ser cualitativa (centrada en juicios y opiniones expertas ante la carencia o escasez de datos) o cuantitativa (fundamentada en la aplicación de principios matemáticos a los datos recolectados). Por su parte, Runes (2018) expresa que una construcción es un proceso mental, mediante el cual se idea el resultado de una serie de actividades.

Para Máttar y Cuervo $(2016$, p.19) "...la construcción de futuros es una tarea colectiva que compete al conjunto de la sociedad. Requiere, ... de la movilización de expertos, científicos y universidades, pero por ningún motivo debe limitarse a los estrechos círculos que estos integran". Es un vocablo vinculado con la estrategia y la prospectiva, entendida por estos autores y por Mera (2014) como una disciplina que tiene como finalidad el diseño de un escenario futuro o desarrollo a corto, mediano y largo plazo, procurando limitar el impacto del azar.

\section{Incertidumbre}

Las expectativas no están exentas de incertidumbre, la cual se presenta cuando no hay seguridad de los resultados, dependiendo esta última, según Newstrom (2011), de la percepción sobre la relación entre esfuerzo, desempeño y retribuciones, lo cual suele verse complicado por el control de terceros (en este caso, representados por el Estado) y de las políticas públicas (Osorio, 2019); constituyendo un elemento relacionado con los estilos gerenciales y los niveles de tolerancia a la ambigüedad, lo cual podría requerir, en el contexto 
actual de las universidades autónomas venezolanas, de lo que Robbins y Coulter (2018,) caracterizan como un estilo analítico y capacidad de tolerancia por parte de la gerencia, para que logren motivar a la comunidad de estas organizaciones.

Esto conlleva a que las autoridades rectorales deban trabajar con una cantidad suficiente de información, para contar con diversas opciones antes de tomar una decisión, así como para estar en posibilidad de demostrar su capacidad de adaptación o ajuste ante situaciones particulares, teniendo en cuenta el mantenimiento de una comunicación eficaz con el personal que hace vida en la universidad, considerado un elemento estratégico de la direccionalidad o liderazgo gerencial por Lussier y Achua (2016).

\section{Proyecto de país}

De acuerdo con Robbins y Coulter (2018), un proyecto es un proceso compuesto por una serie de actividades que cuentan con un inicio y un final precisos, pensados para ser desarrollados en una única oportunidad. Por su parte, Máttar y Cuervo (2016), lo presentan como plan de nación o de gobierno, un término político que contempla la planificación de desarrollo pensada para el progreso de un país, generalmente dirigida desde un gobierno central, agregando que el mismo "... debe servir de hilo conductor, de principio ordenador de las políticas públicas" (Martin, 2005, citado por Máttar y Cuervo, 2016, p.97), lo cual vincula el vocablo con el plan desarrollo-país contemplado en los artículos 2 al 6 de la Ley de Universidades (Consejo Nacional de Universidades, 1970), donde se señala la orientación esperada en el accionar de estas instituciones, como se señala en el siguiente apartado.

\section{Universidad autónoma venezolana}

La Ley de Universidades venezolana vigente (Consejo Nacional de Universidades, 1970), en sus artículos 8 al 10, distingue entre instituciones nacionales y privadas, las describe como autónomas y de conformidad con la Ley de Educación, el Ejecutivo Nacional y el Consejo Nacional de Universidades, puede crear instituciones experimentales. Al respecto, Albornoz (2013), plantea que las universidades nacionales no experimentales han sido reconocidas como organizaciones convencionales 0 autónomas, siendo esta última la opción adoptada para este estudio, representadas, respectivamente por la Universidad Central de Venezuela (UCV), la Universidad de Los Andes (ULA), la Universidad del Zulia (LUZ), la Universidad de Carabobo (UC) y la Universidad de Oriente (UDO).

Como organizaciones sociales, las universidades autónomas venezolanas representan un sistema complejo, entendido, como lo expresan Bolman y Deal (2017), como instituciones donde destaca la convergencia de relaciones humanas e interacción social, mientras la concepción de los cambios se relaciona con una posibilidad cierta y un proceso vinculado con la dinámica organizacional; constituyendo, según Runes (2018), una totalidad estructurada y un sistema unitario dinámico, conformado por varias partes que contemplan un objetivo común.

Desde la perspectiva de Lucas, García y Llano (2013), estas organizaciones constituyen agrupaciones de personas vinculadas con el funcionamiento básico de la sociedad, donde se asumen modelos garantes de su funcionalidad acordes con su contexto, manifestando la necesidad de estudiarlas desde una perspectiva basada en procesos. En este sentido, agregan que su complejidad viene dada por el número de niveles jerárquicos y de unidades de 
apoyo, es decir, por su diferenciación vertical y horizontal, lo cual afecta su flujo comunicacional, un aspecto coincidente con la visión racionalista o estructural de Bolman y Deal (2017).

De conformidad con lo anterior, las universidades autónomas venezolanas están inmersas en un contexto político, económico y social, donde todo proceso gerencial debe enfrentar retos para dar alcance a sus objetivos y cumplir sus funciones, las cuales a su vez deben estar en concordancia con un plan desarrollo-país, como lo establece la vigente Ley de Universidades (Consejo Nacional de Universidades, 1970), cuyos artículos 2 al 6 especifican que estas instituciones están al servicio del país y les corresponde contribuir en la orientación de la vida nacional.

Considerando este basamento legal (Ley de Universidades de 1970), cabe destacar que en los artículos 47 y siguientes, se define la estructuración de las universidades venezolanas, la cual contempla diferentes niveles jerárquicos repartidos entre las diversas Facultades, Escuelas, Institutos y Centros de Investigación que las conforman, acordes con su disciplina de formación, siendo institucionalmente complejas, porque su tamaño, la variedad de programas académicos, el sistema colegiado de decisiones, la cantidad de pasos y consultas que conlleva cada proyecto en la escala jerárquica y el número de servicios ofrecidos, las convierten en entes con un complicado entramado administrativo y funcional.

En este sentido, se da cabida a la definición de Rama (2008), para quien la universidad es una organización social compleja conformada por un sistema donde intervienen aspectos simbólicos (conocimientos, creencias), sociales (individuos, grupos, partidos políticos) y diferentes estructuras engranadas, añadiendo que es una institución reactiva ante las diversas demandas de la sociedad. De acuerdo con planteamientos más recientes de este autor (Rama, 2020), la crisis económica ha afectado no solo sus recursos y la cantidad tanto de docentes como de estudiantes, sino también la seguridad de los campos universitarios.

\section{Aspectos metodológicos}

El estudio desarrollado se enmarcó en el paradigma interpretativo, siguiendo un enfoque cualitativo, realizando aproximaciones sucesivas del fenómeno social seleccionado mediante argumentaciones dialógicas (Corbetta, 2014), a partir de una revisión documental con apoyo en el método hermenéutico y el análisis de contenido (Izcara, 2014), utilizándose la triangulación para otorgarle validez y fiabilidad a la investigación (Álvarez-Gayou, 2003, Taylor, Bogdan y DeVault, 2016).

Respecto a las unidades de observación seleccionadas, estuvieron conformadas por documentos públicos emitidos por autoridades rectorales pertenecientes a las cinco universidades autónomas venezolanas mencionadas con anterioridad, coincidentes en un lapso temporal similar, considerando para ello eventos realizados en fechas de relevancia para estas organizaciones, como actos de grado, aniversarios institucionales y consejos universitarios extraordinarios, emergiendo de la interpretación de sus contenidos las categorías construcción del futuro, incertidumbre y proyecto de país.

Para ello, se realizó una búsqueda de publicaciones institucionales que permitiera acceder a comunicaciones descargables de cada de ellas (ver Cuadro 1), desarrolladas durante el año 2018, momento que se corresponde históricamente con la aprobación de un acuerdo para declarar, durante una sesión de la Asamblea Nacional, la Emergencia Humanitaria Compleja de la educación en el país, con el cual se exhortó al ente gubernamental a cumplir sus 
responsabilidades constitucionales con este sector, una declaratoria a la que se adhirieron paulatinamente las instituciones universitarias autónomas (Prensa Derechos Universitarios, 2018).

El proceso seguido para recopilar la información de interés se inició con el registro de las publicaciones ubicadas, elaborando matrices de observación que permitieron la organización, sistematización y análisis de su contenido, con su consecuente proceso de verificación, relecturas, categorización y codificación. Referente a la categorización, se siguió la propuesta de Taylor, Bogdan y DeVault (2016), consistente en la redacción de una lista de conceptos, temas, interpretaciones, tipologías y proposiciones que surgen de un análisis preliminar y varias lecturas, hasta lograr la depuración de las categorías.

Cuadro 1. Unidades de observación

\begin{tabular}{|c|c|c|c|c|}
\hline $\mathbf{N}^{\circ}$ & $\begin{array}{c}\text { AUTORIDAD } \\
\text { UNIVERSITARIA }\end{array}$ & UNIVERSIDAD & DOCUMENTO & FECHA \\
\hline 1 & Rectora & UCV & $\begin{array}{c}\text { Informe Rectorado UCV } \\
\text { (2018). Palabras de apertura } \\
\text { del evento “Venezuela no se } \\
\text { rinde. Es hora de cambiar", } \\
\text { organizado por Asociación } \\
\text { de Profesores de la UCV. }\end{array}$ & 06 marzo 2018 \\
\hline 2 & Rector & ULA & $\begin{array}{c}\text { Prensa ULA (2018). } \\
\text { Palabras del Rector Mario } \\
\text { Bonucci en los 233 años de } \\
\text { la Universidad de Los Andes }\end{array}$ & 05 abril 2018 \\
\hline 3 & $\begin{array}{c}\text { Consejo Universitario } \\
\text { de LUZ }\end{array}$ & LUZ & $\begin{array}{c}\text { Universidad del Zulia (2018). } \\
\text { Comunicado del CU } \\
\text { Extraordinario sobre la } \\
\text { Crisis Universitaria en LUZ }\end{array}$ & 18 septiembre \\
2018
\end{tabular}

Fuente: Elaboración propia 


\section{Resultados}

La información emitida por las autoridades rectorales de las universidades autónomas, en los documentos analizados, responde a un contexto de crisis institucional, caracterizada como un contenido más político que académico, lineal, dirigido a la comunidad en general (público interno y externo), extendiendo de este modo el alcance de su publicación, la cual puede ser accedida a través de enlaces de periódicos locales, regionales o nacionales y desde las propias Websites de estas instituciones.

Para estos representantes de la gerencia universitaria, resulta imperativo dar a conocer cómo se enfrentan a las limitaciones vivenciadas a diario en cada una de las organizaciones que dirigen, mostrando abiertamente su ideario en pro de un sistema gubernamental opuesto al implementado por el gobierno actual, con el cual mantienen un conflicto abierto y frontal. De este modo, los hallazgos corroboran la caracterización de la universidad planteada por Albornoz (2013), al referirse a la lucha continua de las instituciones autónomas para mantener una sólida presencia en la sociedad venezolana.

\section{Expectativas}

Referente a esta categoría, la unidad de observación 1 (Informe Rectorado UCV, 2018) pone de manifiesto que la universidad no se rinde ante situaciones adversas, adoptando entre sus acciones estratégicas los acuerdos y los manifiestos que expresen sus quejas, reclamos e inquietudes, para mantener su presencia en la cotidianidad nacional. Para la unidad de observación 2 (Prensa ULA, 2018), aunque reconoce que el desarrollo de los acontecimientos recientes no ha sido favorable, resalta la perseverancia de la comunidad universitaria para mantener la universidad en funcionamiento, contribuyendo con el progreso y la prosperidad del país, a pesar de las dificultades.

Por su parte, la unidad de observación 3 (Universidad del Zulia, 2018) expone que las mismas causas por las cuales se ha declarado la universidad en emergencia, amenazan la estabilidad del sistema democrático y constitucional de la nación, haciendo ver que la historia reciente afecta a todas las instituciones vinculadas, agregando que la gerencia pública universitaria está en sesión permanente para recibir, considerar y canalizar las propuestas de reivindicación de los derechos de esa comunidad y de la sociedad en general, así como para conducir esfuerzos destinados a la recuperación de espacios óptimos para la academia y para una vida con dignidad, justicia y bienestar.

Desde la perspectiva de la unidad de observación 6 (Romero, 2018), todos los rectores han sabido afrontar la situación de acuerdo con las circunstancias, sin disminuir su ánimo de superación, enfatizando que sus armas se reafirman en la razón, la verdad y la fuerza, constituyéndose por la fortaleza y la esperanza, lo cual hace innecesario caer en negociaciones para lograr que las personas defiendan la universidad: ésta tiene quien la defienda y ame como institución. Esta opinión es complementada por la unidad de observación 7 (Admin, 2018), al expresar que la adversidad ha sido una constante de la universidad, enfrentada continuamente y en acción conjunta con la capacidad de resiliencia de la comunidad universitaria.

Bajo estas circunstancias, se devela que las expectativas constituyen un componente de la perspectiva de las autoridades universitarias, con posibilidad de marcar una diferencia en el futuro de las universidades autónomas venezolanas. A través de ellas se manifiesta una visión acorde con la situación percibida en el país y de sus intentos por concretar resultados en la 
búsqueda de opciones para lograr la permanencia de estas instituciones, mientras intentan continuar el cumplimiento de sus funciones.

Un resultado que concuerda con el planteamiento de Newstrom (2011) y Runes (2018), al definir las expectativas como la espera de la realización de un acontecimiento basado en motivos razonables y con Warren (2013), al aludir a una atención prospectiva. De igual modo, cuando manifiestan que aspiran a la concreción de una unidad, coinciden con la fórmula de Vroom (Chiavenato, 2017, Lussier y Achua, 2016), donde se menciona la motivación de las personas a desarrollar sus tareas u objetivos a la espera de una recompensa, poniendo de manifiesto vínculos o enlaces entre el esfuerzo y el desempeño, así como entre el desempeño y la recompensa o retribución (Robbins y Coulter, 2018), hasta conseguir la satisfacción por los logros alcanzados, que en este caso se refieren tanto a la recuperación de la universidad autónoma venezolana como del país, de acuerdo con el contenido de la documentación institucional analizada.

En esos documentos, las autoridades rectorales enfatizan la necesidad de unir esfuerzos en el proceso de reconstruir el país, con el fin de retomar la senda del progreso y el desarrollo, recuperando el sistema de gobierno democrático, lo cual consideran parte del compromiso asumido como institución formativa y profesionalizante ante la sociedad venezolana, cuyo basamento se estipula detalladamente en la Carta Magna (denominación alterna de la Constitución de la República Bolivariana de Venezuela, Asamblea Nacional Constituyente, 1999) y en la Ley de Universidades (Consejo Nacional de Universidades, 1970).

Enmarcada la realidad de estas organizaciones en un contexto problemático, las autoridades universitarias concuerdan en realizar un llamado a la unión de voluntades para hacer realidad el sueño compartido de contar con un país en mejores condiciones, venciendo el desánimo y la confusión imperantes mediante el esfuerzo de cada integrante de la universidad autónoma venezolana, buscando apoyarse básicamente en su autonomía. Se ratifica de esta forma la obligación que tienen de responder a las expectativas sociales a través de mejoras en la calidad de su gestión, plasmadas por Dioses et al (2021), a la vez que se coincide con lo expresado por Hernández y Padilla (2019), al caracterizar las expectativas como resultado de valorar lo pertinente y lo posible desde la autopercepción y la situación donde se está inmerso.

\section{Construcción del futuro}

La categoría construcción del futuro es percibida por la unidad de observación 1 (Informe Rectorado UCV, 2018) como el sueño compartido de haber alcanzado una titulación universitaria, con posibilidad de extenderse hacia la construcción de un país que necesita la confluencia de todos para alcanzar el progreso y el desarrollo, lo cual incluye igualmente a la universidad. En consecuencia, las autoridades universitarias realizan un exhorto para dirigir la mirada hacia el interior de cada uno de sus integrantes, partiendo de lo individual, para concretar un reencuentro con los mayores actos de fe, con los compromisos de vida como vía para recobrar la posibilidad de ver hacia el futuro que se desea alcanzar y trabajar por conseguirlo.

Desde su perspectiva, Venezuela merece la oportunidad de lograr que cada uno de sus habitantes pueda mirar con claridad hacia ese futuro soñado. Un futuro de garantías democráticas, de desarrollo, prosperidad y libertades. Un aspecto reforzado por el aporte de la unidad de observación 2 (Prensa ULA, 2018), al señalar la necesidad de desarrollar un trabajo conjunto con todos los venezolanos en procura del retorno a la democracia y la restauración del 
imperio de la ley, dejando clara su esperanza de que quienes han perjudicado a generaciones enteras asuman la responsabilidad de sus actos.

Según esta unidad de observación, los venezolanos llevan ahora una maleta de experiencias por cualquier lugar adonde se dirijan; tras el retorno de la paz, el respeto, la abundancia y la prosperidad, ese equipaje servirá como herramienta para avanzar hacia la calidad de vida que la población merece, la cual se logrará construir entre todos; mencionando además el aprendizaje obtenido, referido a la necesidad de excluir el odio y el resentimiento de las emociones que mueven cada accionar, como vía para lograr unificar esfuerzos en beneficio de la nación.

De igual manera, indica que el camino para levantar el país está escrito, no requiriéndose por tanto de convocar a mesas de diálogo, acuerdos de coexistencia o firmas de pactos, sino centrarse en el contenido de la Constitución Nacional, la Carta Magna de los venezolanos, definida por las autoridades universitarias como el máximo acuerdo social sobre el cual debe evitarse hacer lecturas acomodadas, asumiendo respeto y seguimiento al contenido de sus lineamientos.

Al respecto, indica que en sus acciones no han vacilado en señalar a la dirigencia política la necesidad de mantener un discurso transparente y sin ambigüedades que plantee con claridad el camino a seguir para recuperar el país, sin caudillos ni mesías, solamente con gerentes honestos impulsadores de una verdadera consciencia democrática; añadiendo la unidad de observación 7 (Admin, 2018), que corresponde también a cada egresado universitario demostrar su estirpe, asumiendo su turno de dejar una huella imperecedera en la construcción de una mejor Venezuela para todos.

En virtud de que la construcción de un país y la recuperación de la institución se vincula con su desarrollo y progreso, los hallazgos guardan relación con el artículo 3 de la Ley de Universidades (Consejo Nacional de Universidades, 1970), el cual contempla, como función rectora de estas instituciones, cumplir con la misión de brindar educación, cultura y ciencia a sus estudiantes en formación. Para ello deben planificar actividades dirigidas a la creación, asimilación y difusión del saber mediante procesos de investigación-enseñanza, para lograr una preparación integral de los profesionales requeridos por la nación, un elemento limitado por un financiamiento deficitario.

Las expresiones de las autoridades rectorales concuerdan con el manifiesto de Aula Abierta Venezuela (2020), al hacer referencia al elevado costo cobrado por las presiones generales sobre las instituciones universitarias que, de no ser remediado, puede provocar un impacto negativo tanto en el ámbito de estas organizaciones como en la capacidad misma de la nación para superar la situación de crisis que la caracteriza en la actualidad. Sin embargo y de conformidad con sus planteamientos, la universidad venezolana cuenta con la capacidad y tiene el deber de asumir un rol clave en el proceso de recuperación del país. Un aspecto compartido por Ramírez (2020, p.71), quien enfatiza que "La universidad venezolana se recuperará, ..., ella cuenta con la fuerza moral y el talento humano necesario para emprender esa hermosa tarea".

En este sentido, se ratifican los planteamientos de Marcano (2017), cuando manifiesta que a la gerencia le corresponde asumir y cumplir su responsabilidad, así como estar en capacidad de realizar pronósticos y diseñar estrategias viables, como propone Mera (2014); de igual manera, se corrobora la postura de Sánchez y Cabrera (2021, p.112), quienes enfatizan la necesidad de contar con un liderazgo transformacional compartido, con la finalidad de dar 
alcance a sus objetivos a través de la cooperación y el esfuerzo de todos, internalizando y externalizando el carácter colectivo de esa construcción del futuro (Máttar y Cuervo, 2016).

\section{Incertidumbre}

Para la unidad de observación 2 (Prensa ULA, 2018), en este momento Venezuela vive días marcados por el desánimo, la incertidumbre y la confusión, calificando como inaceptable la situación del país, a pesar de lo cual puede apreciarse el cambio en cada una de las personas como individuos, tal vez más humanos, exhortando a la comunidad universitaria y a la sociedad en general a evitar perder el derecho a soñar y ser felices, exponiendo que una mejor nación nace cada día en sus corazones.

De manera complementaria, la unidad de observación 3 (Universidad del Zulia, 2018) da a conocer la realización de sesiones extraordinarias de Consejos Universitarios, con participación de integrantes gremiales profesorales, estudiantiles y trabajadores de cada institución para analizar la situación de la universidad respecto al cumplimiento de sus funciones fundamentales, considerando el contexto regional y nacional, recordando cómo en enero del año 2018 el cogobierno universitario se declaró en emergencia, ante las continuas distorsiones sociales y económicas que han venido afectando al país, percibiendo la comunidad universitaria el agravamiento de la crisis política, responsable de alterar el funcionamiento de las instituciones del Estado.

Desde su visión, el modelo económico propicia un estado de hiperinflación indetenible, la degradación de las condiciones ya mínimas de la población para el ejercicio de los derechos a la alimentación y la salud, la emigración multitudinaria y creciente de venezolanos de todas las edades y sectores sociales, así como la emergencia que ha causado en todo el país la crisis de servicios esenciales como la electricidad y el transporte público, manifestándose con fuerza en la capacidad de la universidad para organizar actividades correspondientes con sus compromisos de ley.

La unidad de observación 4 (Aular, 2018b) ratifica este panorama, al expresar que en los últimos meses los Consejos Universitarios han recibido cientos de permisos y renuncias tanto de profesores como de empleados de todas las facultades y núcleos, quienes por la situación se van del país en busca de mejores condiciones laborales, a lo cual se une la falta de apoyo gubernamental para llevar a cabo diversos procesos de investigación. Una situación extensible a los estudiantes, señalando que muchos de ellos han abandonado las aulas de clases para cruzar las fronteras con el fin de poder llevar un sustento económico a sus hogares.

Una situación corroborada por la unidad de observación 5 (Primera, 2018), cuando manifiesta que la universidad ha pasado y pasa por circunstancias difíciles, imposiciones externas gubernamentales que afectan su autonomía y repercuten en su desempeño, como es la asignación de un presupuesto que a lo sumo cubre un $20 \%$ de sus requerimientos planificados, quedando ausente lo necesario para el funcionamiento. Una realidad presupuestaria a la cual se suma la severa crisis estructural que vive el país y una hiperinflación que asfixia a todas las instituciones, siendo difícil que alguna planificación pueda ajustarse a lo que califican como volátil y feroz crisis económica.

Desde la perspectiva de la unidad de observación 6 (Romero, 2018), el aniversario de cada universidad llega en medio de una crisis financiera acompañada del éxodo de estudiantes, profesores y empleados que buscan oportunidades en el exterior; culminando esta imagen con 
el aporte de la unidad de observación 7 (Admin, 2018), al expresar que ante la grave crisis política, económica y social que atraviesa el país, los nuevos profesionales deben demostrar su linaje y la formación recibida.

Las exposiciones de estas autoridades universitarias se relacionan con un proceso de revaloración de las personas, que se ha desarrollado con la llegada del nuevo milenio y un enfoque integral humanista, un cambio paradigmático descrito por Bokova (2010) como incertidumbre, debido a la complejidad que caracteriza la realidad de este nuevo período histórico, un aspecto destacado de forma reiterada por esas unidades de observación. En este sentido, coincidiendo con Bolman y Deal (2017), visualizan los cambios como una posibilidad cierta, es decir, como un proceso más de la dinámica organizacional y no como un factor ajeno a ella, que a su vez requiere manifestar un nivel elevado de tolerancia (Robbins y Coulter, 2018) y capacidad de ajuste ante situaciones particulares, apoyado en un sistema comunicacional eficaz (Lussier y Achua, 2016).

De igual manera, se ratifican los planteamientos de Albornoz (2013) y Acosta (2018), cuando expresan que las universidades públicas son las más afectadas por la crisis vivenciada, agregando que esta situación se refuerza por el carácter híbrido que caracteriza al gobierno (Estado) actual, donde se combinan prácticas democráticas y autocráticas, destacando la poca o nula negociación con quienes asumen una postura opuesta a sus directrices, una posición propia de las instituciones universitarias autónomas del país; conforme la argumentación de Osorio (2019), se trata de una situación constante derivada de las políticas tomadas por el gobierno central.

\section{Proyecto de país}

Finalmente, la categoría proyecto de país es descrita por la unidad de observación 2 (Prensa ULA, 2018) como la obligación y destino de la nación a renacer de sus cenizas, destacando en los documentos que el único proyecto nacional aceptable por los venezolanos es uno soportado en instituciones sólidas, un elemento vinculable con el planteamiento de desarrollo y progreso expuesto en los artículos 2 al 6 de la Ley de Universidades (Consejo Nacional de Universidades, 1970).

Sin embargo, en el caso de las universidades autónomas venezolanas, la posición gerencial de estas instituciones es opuesta al plan de desarrollo propuesto por el gobierno actual con su Plan de la Patria, de tendencia socialista (un proyecto ambiguo, de acuerdo con Osorio, 2019). Ante un contexto de incertidumbre político-económica, sus autoridades manifiestan que el accionar gerencial universitario se enfrenta a condiciones adversas, las cuales han pasado a formar parte de su cotidianidad, mientras dan continuidad a sus intentos de alcanzar sus objetivos y cumplir sus funciones inherentes.

Un aspecto que coincide con la situación de asfixia financiera descrita por Aula Abierta Venezuela (2020, p.3), al enfatizar la dependencia íntegra del presupuesto universitario de la asignación presupuestaria que realiza el Estado, agregando cómo "Esto deja a las universidades públicas abiertas a la politización de las decisiones de financiación y puede exponer a las universidades que se consideran ideológicamente no alineadas con el gobierno gobernante a un déficit de financiación".

De manera simultánea, se ratifica el planteamiento de Pérez y Moreno (2017), quienes destacan el esfuerzo de las autoridades rectorales para lograr el mantenimiento de la calidad en 
el cumplimiento de las funciones básicas de las organizaciones que dirigen, como demostración de acatamiento de sus respectivas misiones y visiones institucionales, lo cual puede significar que, para lograrlo, deban asumir la propuesta de Marcano (2017), referida a la prioridad de lograr una transformación universitaria, o aquella de Marín (2020), vinculada con la búsqueda de cooperación a través del establecimiento de acuerdos estratégicos con universidades iberoamericanas, como muestra de una visión ampliada para lograr un planificado progreso nacional (Máttar y Cuervo, 2016).

Gráfico 1. Expectativas de las autoridades rectorales sobre la universidad autónoma

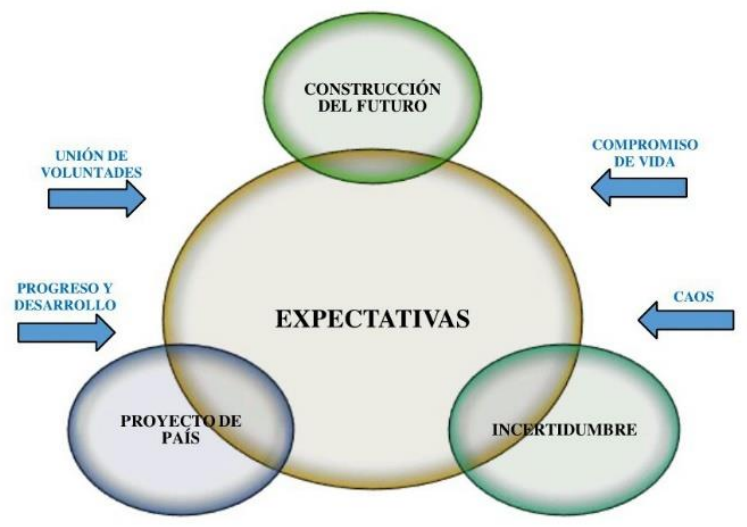

Fuente: Elaboración propia

El Gráfico 1 ilustra la perspectiva de las autoridades rectorales. De este modo, se revela que las expectativas constituyen un estado de esperanza, caracterizándose, de acuerdo con los hallazgos, como la necesidad de lograr la unión de la comunidad para encaminar esfuerzos en el proceso de reconstrucción de la universidad y del país. En este sentido, se daría cumplimiento a la contribución que debe hacer la universidad autónoma venezolana en pro del progreso y el desarrollo de la nación, de conformidad con lo expresado en la Constitución Nacional y la Ley de Universidades, en vinculación con las categorías construcción del futuro, incertidumbre y proyecto de país.

Respecto a la construcción de futuro, es conceptualizado como un sueño compartido que, para convertirse en realidad, requiere la unificación de los esfuerzos de la comunidad universitaria y de la sociedad venezolana en general. El inicio de esta tarea contempla una revisión de la individualidad de cada uno, incluyendo el compromiso de vida particular para viabilizar el camino a seguir, especificando como base la recuperación de la democracia y la restauración de los preceptos legales establecidos y todavía vigentes, constituyendo la Constitución Nacional venezolana el mejor ejemplo de acuerdo social.

En cuanto a la incertidumbre, es comprendida como una situación caracterizada por la confusión o caos ocasionado por la distorsión socioeconómica del contexto nacional, producida a su vez por las políticas implementadas por el gobierno central y el modelo 
económico asumido. La realidad-país se describe como un proceso hiperinflacionario indetenible, una emigración en ascenso de la población en general, una disminución de la calidad de vida de la comunidad, una crisis política agravada y una trasformación severa del funcionamiento tanto de las instituciones gubernamentales como de las universidades autónomas venezolanas.

Finalmente, referente al proyecto de país, es definido como un renacimiento de la nación, centrado en organizaciones sólidas, cuyas actividades sean coherentes con su institucionalidad y la normatividad regulada por la legislación vigente, para lograr la meta de encaminarla en un plan político-económico consistente con las necesidades de desarrollo y progreso de la población en general, siguiendo las pautas establecidas en la Carta Magna y en la Ley de Universidades.

\section{Conclusiones}

La revisión de los documentos seleccionados posibilitó dar alcance al objetivo de describir las expectativas sobre la universidad autónoma venezolana desde la perspectiva de su gerencia, las cuales son entendidas como un estado esperanzador, que requiere lograr la unión de quienes han hecho y hacen vida en estas instituciones para encaminar esfuerzos en el proceso de reconstrucción del país. Como sueño compartido, el anhelo de construir un futuro requiere de un esfuerzo mancomunado y un compromiso común que, a pesar de las diferencias, permita alcanzar esa meta.

Un esfuerzo que precisa igualmente considerar la incertidumbre como un factor de riesgo, el cual debe ser cuidadosamente administrado para evitar caer en ofrecimientos poco viables. La situación-país actual requiere pasos firmes y seguros para lograr convencer a quienes duden, que se trata de actuar estratégicamente con base en la experiencia y el conocimiento para lograr revertir con efectividad el panorama reinante, contando de ese modo con mayores oportunidades de trabajar como unidad, manteniendo al mismo tiempo coherencia con la institucionalidad de la universidad y la legislación bajo la cual se ampara.

Las autoridades rectorales manifiestan, de este modo, estar en capacidad de enfrentar la problemática reinante, descrita como caos, buscando fortalecerse con el apoyo de la comunidad. Se imaginan un mejor futuro, viable con el esfuerzo de cada uno de los integrantes del país, invitando a extender el compromiso de vida individual hacia la recuperación social, económica y política venezolana, como producto de un ejercicio de introspección. Desde su perspectiva, tanto la universidad autónoma nacional como el país, pueden mejorar su situación y lograr la recuperación de garantías democráticas, progreso, libertad y desarrollo.

Los hallazgos permiten enfatizar la voluntad, deseo y esperanza de las autoridades universitarias por recuperar la nación venezolana, a través de aspectos como la construcción del futuro, manejo de la incertidumbre y un proyecto de país. Reiteran en ellos el requerimiento de unir esfuerzos, considerándolo un deber como institución formativa y profesionalizante, de conformidad con las pautas señaladas tanto en la Carta Magna como en la legislación universitaria.

De conformidad con lo anteriormente expresado, se concluye que las autoridades rectorales de las universidades autónomas venezolanas cuentan con las herramientas necesarias para superar su crisis institucional, experimentada temporalmente antes de la aparición del Covid-19, pero también deben considerar su deber el fortalecimiento de las 
comunicaciones con la comunidad interna y externa a ellas, en pro de la fluidez y transparencia de la información contentiva de sus planes si de verdad aspiran lograr el apoyo y la unificación de voluntades requeridos para recuperar su prestigio.

La tarea de la gerencia de estas instituciones no es sencilla. Se enfrentan de forma simultánea a diversas situaciones internas y externas, con diferentes grados de afectación en su accionar cotidiano. Su complejidad de organizacional deriva de la diversidad estructural de su interior, la disparidad estratégica de sus distintos gerentes, la variedad de las demandas que recibe y la predisposición propia de la extensa especialidad capaz de ofrecer, pudiendo describirse como una forma de asociación creada a partir de un entramado legislativo que dictamina su normativa organizativa y de funcionamiento, un elemento esencial que no pueden perder de vista.

\section{Referencias Bibliográficas}

Acosta, Yorelis (2018). Sufrimiento psicosocial del siglo XXI: Venezuela y la revolución. Revista de Psicología,19. Bolivia. (Pp.111-134).

Admin. (2018). UDO cuenta con 500 nuevos egresados. Extraído de https://elluchador.info/web/2018/11/09/udo-cuenta-con-500-nuevos-egresados/

Albornoz, Orlando. (2013). La universidad ¿ reforma o experimento? El discurso académico contemporáneo según las perspectivas de los organismos internacionales: los aprendizajes para la universidad venezolana y latinoamericana. UNESCO-IESALC, Venezuela.

Álvarez-Gayou, Jurgenson. (2003). Cómo hacer investigación cualitativa. Fundamentos y metodología. Paidós, México.

Asamblea Nacional Constituyente. (1999). Constitución de la República Bolivariana de Venezuela. Gaceta Oficial de la República de Venezuela 36860. Venezuela.

Aula Abierta Venezuela. (2020). El declive de la universidad venezolana. Extraído de SAR-Freeto-Think-2020-Venezuela-Spanish.pdf (scholarsatrisk.org)

Aular, Judith. (2018a). Reinvención de la universidad. Extraído de http://www.viceacademico.luz.edu.ve/index.php?option=com_content\&task=view\&id=20 64\&ltemid=202

Aular, Judith. (2018b). LUZ fortalece sus raíces en sus 127 años. Extraído de http://www.laverdad.com/opinion/145971-luz-fortalece-sus-raices-en-sus-127-anos.html

Aular, Judith. (2019). Compromiso con LUZ. Extraído de https://noticiaaldia.com/2019/03/compromiso-con-luz-dra-judith-aular-de-duran/

Bokova, Irina. (2010). Un nuevo humanismo para el siglo XXI. Recuperado de https://unesdoc.unesco.org/ark:/48223/pf0000189775 spa

Bolman, Lee y Deal, Terrence. (2017). Reframing organizations: artistry, choice and leadership. John Wiley and Sons, United States of America.

Cárdenas, Leomara. (2019). Profesores de la UC asumirán paro escalonado. Extraído de http://cronica.uno/profesores-de-la-uc-asumiran-paro-escalonado/

Chiavenato, Idalberto. (2017). Comportamiento organizacional. La dinámica del éxito en las organizaciones. McGraw-Hill, España.

Consejo Nacional de Universidades. (1970). Ley de Universidades. Gaceta Oficial de la República de Venezuela 1.429. Venezuela. 
Corbetta, Piergiogio. (2014). Metodologia e tecniche della riserca sociale. IIMulino, Italia.

Dioses, Nelly; Morales, Humberto; Díaz, Doris y Vásquez, Antenor. (2021). Nivel de satisfacción en la formación de calidad a nivel universitario. Telos: revista de Estudios Interdisciplinarios en Ciencias Sociales, 23 (2), Venezuela. (Pp.247-266).

DOI: www.doi.org/10.36390/telos232.04

Drucker, Peter. (2004). La Gerencia en la sociedad futura. Norma, Colombia.

García-Arocha, Cecilia. (2018). Discurso de la Rectora Cecilia García Arocha en Acto del Aula

Magna. Extraído de http://ucvnoticias.ucv.ve/?p=68615

Hernández, Oliveira y Padilla, Laura. (2019). Expectativas de los estudiantes hacia la educación superior: influencia de variables familiares, personales y escolares. Sociológica, 34

(98), México. (Pp.221-251). Extraído de 2007-8358-soc-34-98-221.pdf (scielo.org.mx) Informe Rectorado UCV. (2018). Palabras de apertura del evento "Venezuela no se rinde. Es hora de cambiar". Extraído de http://www.ucv.ve/fileadmin/user_upload/rectorado/InformesSemanales/INFORMEREC TORAD013 03 18.pdf

Izcara, Simón. (2014). Manual de investigación cualitativa. Fontamara, México.

Lucas, Antonio, García, Pablo y Llano, Sergio. (2013). Sociología de las organizaciones. Influencia de las tecnologías de la información y la comunicación. Fragua, España. Lussier, Robert y Achua, Christopher. (2016). Liderazgo. Teoría, aplicación y desarrollo de habilidades. Cengage, México.

Marcano, Josseilin. (2017). Análisis crítico de la gerencia y transformación en la educación universitaria, desde diversos postulados. Revista Scientific, Vol.2, Edición Especial. Venezuela. $\quad$ (Pp.275-294). DOl: $\quad$ https://doi.org/10.29394/scientific.issn.25422987.2017.0.0.15.275-294

Marín, Freddy. (2020). Resignificar la praxis educativa en tiempos de incertidumbre: un desafío para las organizaciones sociales. Revista de Ciencias Sociales (RCS FCES LUZ), 26
(2)
(número
especial),
Venezuela
(Pp.9-13).
DOI:

https://doi.org/10.31876/rcs.v26i0.34140

Máttar, Jorge y Cuervo, Mauricio. (2016, compiladores). Planificación y prospectiva para la construcción de futuro en América Latina y el Caribe. Textos seleccionados 20132016.

https://www.cepal.org/sites/default/files/publication/files/40623/S1600345 es.pdf

Mera, Carlos. (2014). Pensamiento prospectivo: visión sistémica de la construcción de futuro. Análisis. Revista Colombiana de Humanidades, 46 (84), Colombia. (Pp.89-104).

Newstrom, John. (2011). Comportamiento humano en el trabajo. McGraw-Hill, México.

Osorio, Leonardo. (2019). El socialismo del siglo XXI y la crisis de la sociedad venezolana.

Telos: revista de Estudios Interdisciplinarios en Ciencias Sociales, 21 (1), Venezuela. (Pp.33-60). DOI: www.doi.org/10.36390/telos211.04

Pérez, Giovanni y Moreno, Zahira. (2017). Constructo teórico sobre gerencia universitaria. Criterio Libre, 15 (26), Colombia. (Pp.23-42). DOI: https://doi.org/10.18041/19000642/criteriolibre.2017v15n26.1030

Prensa Derechos Universitarios. (2018). Universidades venezolanas declaradas en emergencia humanitaria compleja. Extraído de Universidades venezolanas declaradas en Emergencia Humanitaria Compleja - Derechos Universitarios 
Prensa ULA (2018). Palabras del rector Mario Bonucci en los 233 años de la Universidad de Los Andes. Extraído de http://prensa.ula.ve/2018/04/05/palabras-del-rector-mario-bonuccien-los-233-a\%C3\%B1os-de-la-universidad-de-los-andes

Primera, Marlene. (2017). El rol universitario frente a la realidad del país. Extraído de http://www.secretaria.luz.edu.ve/index.php?option=com content\&task=view\&id=1053\&l temid $=205 \mathrm{v}$

Primera, Marlene. (2018). Reapertura de LUZ. Extraído de Diario La Verdad - Reapertura de LUZ

Rama, Claudio. (2008). La complejidad de las organizaciones universitarias. Seminario Permanente. Comisión de Transición de la Universidad para discutir y consensuar sobre la reforma en curso de la universidad. Tegucigalpa, Honduras. Extraído de https://es.slideshare.net/claudiorama/la-comlejidad-de-la-organizaciones-universitarias

Rama, Claudio. (2020). Mirada a la educación superior en Venezuela. Universidades, 71 (83), México. (Pp.7-16). DOI: https://doi.org/10.36888/udual.universidades.2020.83.72

Ramírez, Tulio. (2020). La universidad autónoma venezolana y su lucha por sobrevivir al socialismo del siglo XXI. Cinco propuestas para su rescate. Universidades, 71 (83). México. (Pp.53-72). DOI: https://doi.org/10.36888/udual.universidades.2020.83.76

Robbins, Stephen y Coulter, Mary. (2018). Administración. Pearson, México.

Romero, Tibisay. (2018). Universidad de Carabobo celebró 60 años de su reapertura. Extraído de http://elestimulo.com/blog/universidad-de-carabobo-celebro-60-anos-de-sureapertural

Runes, Dagobert. (2018). The dictionary of philosophy. Creative Media Partners, LLC., USA. Sánchez, Roemith y Cabrera, Isaida. (2021). Gerencia y responsabilidad social: construcción colectiva desde la complejidad y la acción comunicativa. Telos: revista de Estudios Interdisciplinarios en Ciencias Sociales, 23 (1), Venezuela. (Pp. 100-114). DOI: www.doi.org/10.36390/telos231.08

Taylor, Steven, Bogdan, Robert y DeVault, Marjorie. (2016). Introduction to qualitative research method. (4th edition). John Wiley \& Sons, Inc., USA.

Universidad del Zulia. (2018). Comunicado del CU Extraordinario sobre la crisis universitaria en LUZ.

Extraído de

http://luz.edu.ve/index.php?option=com_content\&view=article\&id=2515:comunicadodel-cu-extraordinario\&catid $=85 \&$ ltemid $=489$

Warren, Howard. (2013, compilador, editor). Diccionario de psicología. Fondo de Cultura Económica, México. 